\title{
Efficient hyper-parameter determination for regularised linear BRDF parameter retrieval
}

Article

Accepted Version

Zobitz, J. M., Quaife, T. and Nichols, N. K. (2020) Efficient hyper-parameter determination for regularised linear BRDF parameter retrieval. International Journal of Remote Sensing, 41 (4). pp. 1437-1457. ISSN 1366-5901 doi:

https://doi.org/10.1080/01431161.2019.1667552 Available at https://centaur.reading.ac.uk/86107/

It is advisable to refer to the publisher's version if you intend to cite from the work. See Guidance on citing.

To link to this article DOI: http://dx.doi.org/10.1080/01431161.2019.1667552

Publisher: Taylor \& Francis

All outputs in CentAUR are protected by Intellectual Property Rights law, including copyright law. Copyright and IPR is retained by the creators or other copyright holders. Terms and conditions for use of this material are defined in the End User Agreement.

www.reading.ac.uk/centaur 
Central Archive at the University of Reading

Reading's research outputs online 


\title{
Efficient hyper-parameter determination for regularised linear BRDF parameter retrieval
}

\author{
J. M. Zobitz ${ }^{\mathrm{a}}$, T. Quaife ${ }^{\mathrm{b}}$, and N. K. Nichols ${ }^{\mathrm{c}}$ \\ ${ }^{a}$ Department of Mathematics, Statistics, and Computer Science, Augsburg University, 2211 \\ Riverside Avenue, Minneapolis, MN, USA; ${ }^{\mathrm{b}}$ National Centre for Earth Observation, \\ Department of Meteorology, University of Reading, Reading, RG6 6BB, UK; ' Department \\ of Mathematics, University of Reading, Reading, RG6 6BB, UK.
}

\section{ARTICLE HISTORY}

Compiled August 30, 2019

\begin{abstract}
Linear kernel driven models of the surface Bidirectional Reflectance Distribution Function (BRDF) are valuable tools for exploiting Earth observation data acquired at different sun-sensor geometries. Here we present a method that efficiently determines linear BRDF model weights using Tikhonov smoothing where the smoothing parameter $\lambda$ is determined via a Generalised Singular Value Decomposition with the root mean square error prescribed depending on the MODIS band. We applied this method to twenty-six different deciduous broadleaf sites across an entire year using the MODIS Terra and Aqua reflectance data products. Kernel weights and white sky albedo derived from this GSVD method were generally consistent with those provided by the MCD43 data products. The GSVD derived results had less sample variability compared to the MCD43 data products, attributable to the assumed smoothness between kernel weights in the Tikhonov smoothing method. The GSVD technique consistently outperforms MCD43 in the reconstruction of observed MODIS reflectance data, of which retrievals from this method will do a better job of estimating albedo and normalising data to specified geometries.
\end{abstract}

\section{KEYWORDS}

bidirectional reflectance; data analysis; reflectance; regularisation; generalised singular value decomposition; Taylor diagrams

\section{Introduction}

Since their inception several decades ago, modern wide-swath remote sensors such as MODIS Terra and Aqua provide an unprecedented view of biological and ecological processes across large landscapes (Cleugh et al. 2017; Mu et al. 2013; Guay et al. 2014; Kussul et al. 2015; Mu et al. 2013). Data products provided by these sensors have broad applications for terrestrial ecosystems. Some of these applications include (1) validation and prediction of landscape primary productivity (Turner et al. 2003, 2006; Hilker et al. 2008; Hufkens et al. 2012; Pan et al. 2014), (2) determining the timing of phenological changes such as spring green up or disturbance (Soudani et al. 2008; Hufkens et al. 2012; Garonna et al. 2014; Hirschmugl et al. 2017), or (3) quantifying biophysical measurements (e.g surface temperature, emissivity, albedo) (Hashimoto 
et al. 2008; Li et al. 2013; Qu et al. 2014). Beyond ecological and biological contexts, such remote sensing data can also be applied in near real-time applications such as drought, public health, or disaster management (Mu et al. 2013; Kansakar and Hossain 2016; Mahecha et al. 2017).

For any given point on the surface of the Earth wide swath sensors such as MODIS provide data at a variety of sun-sensor geometries. Consequently there is a signal in those data from the Bi-directional Reflectance Distribution Function (BRDF) of the surface. For many applications it is important to correctly account for this effect, either to normalize observations to a common geometry or to exploit this information to estimate onward variables such as albedo. The MCD43 data products are derived from MODIS Terra and Aqua sensors, which in turn serve as inputs to several other downstream products. The data products transform surface reflectance $\rho$ at wavelength $\xi$, given viewing and illumination geometries $\Omega^{\prime}$ and $\Omega$, to express them as a linear transformation of different scattering processes with a series of kernels $K_{j}$ :

$$
\rho\left(\Omega, \Omega^{\prime}, \xi\right)=\sum_{j=1}^{3} f_{j}(\xi) K_{j}\left(\Omega, \Omega^{\prime}\right) .
$$

The variable $j$ indexes the different kernels (isotropic, volumetric, and geometric respectively). The kernels $K_{j}$ represent photon scattering from isotropic, volumetric, and geometric processes and quantify the variation of reflectance with the viewing and illumination geometry, and $f_{j}$ denotes the kernel weights for the contribution of each kernel at a given wavelength (Roujean, Leroy, and Deschamps 1992; Wanner et al. 1997; Strahler and Muller 1999; Lucht and Schaaf 2000). In the MCD43 product the isotropic kernel has a value of unity and the volumetric and geometric kernels are the so-called Ross-Thick and Li-Sparse models (Wanner et al. 1997). When kernel weights $f_{j}$ in Equation (1) are determined via a given set of $m$ reflectance measurements across $t$ time periods, Equation (1) can be succinctly described as a matrix equation:

$$
\rho=\mathbf{K} f
$$

where $\mathbf{K}$ is a matrix of the kernels $K_{j}, \boldsymbol{\rho}$ is a vector of $m$ reflectance values and $\boldsymbol{f}$ is a vector of kernel weights of length $n$, which for this study equals 1095 (three kernel weights times the number of days in a year). Since Collection 6 of the MODIS data processing a 16-day sliding window is applied where the window is moved one day per retrieval thus generating a daily product. Mathematically Equation (2) does not have a unique solution when $\mathbf{K}$ is not full rank.

There have been a number of developments to kernel-driven BRDF models over recent years. Important examples include the development of the $\mathrm{Li}$-Transit kernel (Gao et al. 2000) which selects between the Li-Sparse and Li-Dense kernels at the sun-sensor geometry transition point at which the two kernels are equivalent. This provides improved fitting for dense canopies compared to the Li-Sparse kernel that is used in the operational algorithm. A snow kernel has recently been proposed (Jiao et al. 2019) to better account for strong forward scattering that is present in snow. This results in the introduction of an additional free parameter into the inversion, for which the authors provide a default value that can be used for applications where tuning a non-linear parameter might not be practical. A modification to improve the hotspot 
representation in the Ross-Thick kernel also exists (Jiao et al. 2016) and closely follows the modification proposed by Chen and Cihlar (1997).

However, the actual kernels used in the operational MCD43 product have not changed since the publication of the Algorithm Theoretical Basis Document. The inversion algorithm, on the other hand, has been updated. The original product used a 16-day window, which was moved forward in time by eight days for each inversion and used standard least squares. There were separate data products for Terra and Aqua MODIS (MOD43 and MYD43 respectively). The current algorithm combines data from both sensors and weights data to favour the centre of the window more than those on either side.

One alternative to the 16-day sliding window approach relies on mathematical assumptions based on the temporal structure of kernel weights $f$. A reasonable assumption in many cases is that the kernel weights relative to time should be smooth. The approach proposed in Quaife and Lewis (2010) introduced a first order smoothing matrix $\mathbf{B}$ which when considered with Equation (2) led to the following solution for $f$ :

$$
\boldsymbol{f}=\left(\mathbf{K}^{\mathrm{T}} \mathbf{K}+\lambda^{2} \mathbf{B}^{\mathrm{T}} \mathbf{B}\right)^{-1} \mathbf{K}^{\mathrm{T}} \boldsymbol{\rho},
$$

where in Equation 3 the hyperparameter $\lambda$ controls the amount of resulting smoothness in the BRDF kernel weights $\boldsymbol{f}$, and $\mathrm{T}$ represents matrix transpose. This approach is more commonly known as Tikhonov smoothing (Tikhonov 1977). The approach presented in Quaife and Lewis (2010) allowed for a large time window (e.g. K and $\mathbf{B}$ contained information for an entire year to estimate daily $\boldsymbol{f}$ ) and the recovery of kernel weights in domains that exhibited a large degree of temporal variability.

The Tikhonov smoothing approach can easily accommodate multiple daily observations in the matrix $\mathbf{K}$ (as in the case when data from the MODIS Terra and Aqua satellites are used together), with two key caveats. First, Equation (3) requires parameterization of $\lambda$ for each MODIS spatial pixel; it is unknown whether the same value of $\lambda$ can be applied across different spatial domains. Second, Equation (3) requires matrix inversion, which may become computationally prohibitive as the size of the matrix increases (Golub and Van Loan 2014).

The goal of this manuscript is to make more robust the approach presented by Quaife and Lewis (2010), focusing on three key objectives: (1) reformulate the Tikhonov smoothing presented in Quaife and Lewis (2010) as a constrained least squares problem in order to determine the hyperparameter $\lambda ;(2)$ corroborate our estimates of the kernel weights $f$ and associated derived products to existing MODIS data products on a daily timescale; and (3) provide recommendations for selection of values of $\lambda$ to allow for the broader applicability of the least squares and optimization methods across large spatial and temporal domains. For our analysis we select sites with a distinct phenological signal, co-located with towers from the FLUXNET database.

\section{Methods}

\subsection{Site Selection \& Data Processing}

We selected MODIS pixels located in deciduous broad-leaf forests that contained eddy covariance towers monitored in the FLUXNET database. (www.fluxdata.org). 
The sites selected spanned a range of latitudes and longitudes across seven different continents (see Figure 1 and Table 1) and the use of deciduous species means that the sites will exhibit temporally variability with which to test the retrievals.

[Figure 1 about here.]

[Table 1 about here.]

We utilised three different MODIS data products, accessed via the AppEEARS web service (https://lpdaacsvc.cr.usgs.gov/appeears/, AppEEARS Team (2018)). The primary data for analysis was daily $500 \mathrm{~m}$ MODIS Terra (MOD09GA, Vermote and Wolfe (2018a)) and MODIS Aqua (MYD09GA, Vermote and Wolfe (2018b)) surface reflectance ( $\boldsymbol{\rho}$ in Equation (1)) and solar and viewing azimuth and zenith angles to compute kernels $K_{j}$ in Equation (1), using equations developed in Strahler and Muller (1999); Lucht and Schaaf (2000). We utilised all seven MODIS bands in the analysis, confining our analysis to measurements made in 2017.

We compare our estimated daily BRDF kernel weights to those provided by the MCD43A1 data product (combined Terra+Aqua, Schaaf and Wang (2018a)). The MCD43A1 data product determines kernel weights from a rolling 16 day window centered on the observation day. (https://lpdaac.usgs.gov/dataset_discovery/ modis/modis_products_table/mcd43a3_v006)

Our derived BRDF kernel weights are utilised to compute white-sky albedo as a linear combination of the kernel weights (Strahler and Muller 1999; Lucht and Schaaf 2000). We also compare our white-sky albedo to the MCD43A3 data product (Schaaf and Wang 2018b).

All data products were filtered using the MODLAND "good" quality assurance flags for both the data quality and state flags. For the MOD09GA data product there are seven data quality flags (one for each MODIS band) and eight different state flags. For a given site and time, a reflectance measurement passed QA if all seven data quality flags and all eight state flags are good. This strict QA filtering was done to avoid different dimensioned $\mathbf{K}$ matrices for each MODIS band. Figure 2 displays the daily temporal coverage for each of the sites listed in Table 1, along with the number of unique days utilised in building the matrix of kernel values, $\mathbf{K}$. We applied the same quality assurance protocols when comparing our results to other MODIS products.

[Figure 2 about here.]

\subsection{Formulation of the constrained least squares problem}

We formulate Equation (2) as an equality-constrained least squares problem:

$$
\text { minimize }\|\mathbf{B} \boldsymbol{f}\| \text { such that } \frac{\|\boldsymbol{\epsilon}\|}{\sqrt{n}}=\delta
$$

where $\boldsymbol{\epsilon}=\mathbf{K} \boldsymbol{f}-\boldsymbol{\rho}$ is the residual vector, $\delta$ a user-defined tolerance for the residual norm (described later), and the matrix $\mathbf{B}$ is a square, rank-deficient smoothing matrix (here we assume it is a first order difference matrix). The quantity $\frac{\|\boldsymbol{\epsilon}\|}{\sqrt{n}}$ is equivalent to the root mean square error (RMSE).

To solve this constrained least squares problem, we consider the Tikhonov smoothing equation for the BRDF kernel weights (Tikhonov 1977) (which is Equation (3) written 
without the inverse):

$$
\left(\mathbf{K}^{\mathrm{T}} \mathbf{K}+\lambda^{2} \mathbf{B}^{\mathrm{T}} \mathbf{B}\right) \boldsymbol{f}=\mathbf{K}^{\mathrm{T}} \boldsymbol{\rho} .
$$

As previously described Equation (5) is a regularised equation that can be applied to solve a rank-deficient least squares problem. In order to determine our solution $f$ (and also $\|\mathbf{B} \boldsymbol{f}\|$ or $\|\boldsymbol{\epsilon}\|$ ) from Equation (5) the parameter $\lambda$ must be specified. For a unique determination of the BRDF kernel weights $f$ from Equation (5) we have made the assumption that the rank of the matrix $\left[\begin{array}{ll}\mathbf{K}^{\mathrm{T}} & \mathbf{B}^{\mathrm{T}}\end{array}\right]^{\mathrm{T}}$ equals $n$, the number of components in $\boldsymbol{f}$; by direct calculation we find this is true for all the sites studied here. We use this formulation to determine daily kernel weights $f$. Over the course of the year $\boldsymbol{f}$ has 1095 components, which is 365 times the number of kernels (isotropic, volumetric, or geometric).

When $\lambda$ needs specification (which is the case for this study) one approach is to consider $\|\mathbf{B} \boldsymbol{f}\|$ and $\|\boldsymbol{\epsilon}\|$ as a parametric curve in $\lambda$ that is monotonically decreasing (termed the $L$-curve, see Figure 5; Hansen (1992); Hansen and O'Leary (1993)). A choice of $\lambda$ represents the weighted average between a solution $f$ that minimizes the residual norm ( $\|\boldsymbol{\epsilon}\|, \lambda$ near 0$)$ or the solution norm ( $\|\mathbf{B} \boldsymbol{f}\|, \lambda$ infinite). To solve Equation (4) we apply Morozov's Discrepancy Principle (Hansen 1992, 1996), which selects the point on the $L$-curve where $\|\boldsymbol{\epsilon}\|=\sqrt{n} \delta$. While the level assigned to $\delta$ can be arbitrary, we fix $\delta$ to be the typical theoretical accuracy for each MODIS band (see Table 2, from values published by the Land Surface Reflectance Science Computing Facility http://modis-sr.ltdri.org/pages/validation.html).

[Figure 3 about here.]

[Table 2 about here.]

\subsection{The Generalised Singular Value Decomposition}

The selection of $\lambda$ to solve Equation (4), and by association, the BRDF kernel weights $\boldsymbol{f}$, varies for each MODIS band. Iteratively determining the value of $\lambda$ via matrix inversion of Equation (5) is computationally inefficient over large time domains. Here we outline an efficient way to determine $\lambda$ through the application of the Generalised Singular Value Decomposition (GSVD, Bai (1992); Golub and Van Loan (2014)) to Equation (5). The GSVD is similar to the singular value decomposition, with the goal of diagonalizing a pair of matrices.

The GSVD computes a matrix decomposition of $\mathbf{K}$ and $\mathbf{B}$ so that $\mathbf{K}=\mathbf{U} \mathbf{\Sigma}\left[\begin{array}{ll}\mathbf{0} & \mathbf{R}\end{array}\right] \mathbf{Q}^{\mathrm{T}}$ and $\mathbf{B}=\mathbf{V M}\left[\begin{array}{ll}\mathbf{0} & \mathbf{R}\end{array}\right] \mathbf{Q}^{\mathrm{T}}$. The matrices $\mathbf{U}, \mathbf{Q}, \mathbf{V}$ are orthogonal. The non-singular matrix $\mathbf{R}$ is upper triangular. The matrices $\boldsymbol{\Sigma}$ and $\mathbf{M}$ are diagonal non-increasing matrices with entries bounded between zero and unity. The ratio of corresponding diagonal entries in $\boldsymbol{\Sigma}^{\mathrm{T}} \boldsymbol{\Sigma}$ to $\mathbf{M}^{\mathrm{T}} \mathbf{M}$ form what are called generalised singular values of $\mathbf{K}$ and $\mathbf{B}$ (Hansen 1996). The specific forms of $\boldsymbol{\Sigma}$ and $\mathbf{M}$ depend on the rank of the matrices $\mathbf{K}, \mathbf{B}$, and $\left[\begin{array}{ll}\mathbf{K}^{\mathrm{T}} & \mathbf{B}^{\mathrm{T}}\end{array}\right]^{\mathrm{T}}$. For the sites we examined (and by association the construction of the matrices $\mathbf{K}, \mathbf{B}$, and $\left[\begin{array}{ll}\mathbf{K}^{\mathrm{T}} & \mathbf{B}^{\mathrm{T}}\end{array}\right]^{\mathrm{T}}$ ), the application of the GSVD leads to a diagonalized problem where we can uniquely determine the parameter $\lambda$ and the BRDF kernel weights $\boldsymbol{f}$.

An advantage to applying the GSVD is that the kernel weights $f$ can be expressed via linear combinations of the GSVD matrices. Similarly, the residual and 
solution norms can also be easily computed via linear combinations of the GSVD matrices (Hansen 1996). We outline the specific matrix decompositions leading to the determination of $\boldsymbol{f},\|\mathbf{B} \boldsymbol{f}\|$ and $\|\boldsymbol{\epsilon}\|$ in the Supplemental data.

We apply LAPACK algorithms to compute the GSVD from $\mathbf{K}$ and $\mathbf{B}$ (Anderson et al. 1999). We apply a bisection method to determine the value of $\lambda$ where $\|\boldsymbol{\epsilon}\|=\sqrt{n} \delta$. In the Supplemental data we provide pseudocode to implement $\|\boldsymbol{\epsilon}\|,\|\mathbf{B} \boldsymbol{f}\|$, kernel weights $\boldsymbol{f}$, and the bisection method.

\subsection{Laplace formulation to determine kernel weights}

We also estimated kernel weights assuming the residuals $\boldsymbol{\epsilon}$ followed Laplace (double exponential) distribution. This approach is similar to the current algorithm behind the MCD43A1 data product (Wang et al. 2018). Kernel weights are estimated using data from a sixteen-day sliding window, with the kernel weights for a day of interest to be day 9 of the sliding window. We require a minimum of seven days worth of data in the window to determine the kernel weights. We denote kernel weights estimated from this method as "Laplace".

\subsection{Algorithm Cross-Validation on Subsetted Data}

To validate the computational approaches utilized we selected the FLUXNET site with the largest number of represented days (195 days, site IT-CA1, Figure 2) and performed a modified cross-validation approach to estimate BRDF kernel weights for the GSVD and Laplace methods. The reflectance data were randomly sampled to a certain percentage $(25 \%, 50 \%, 75 \%$, and $100 \%)$ of the 195 days to generate a smaller vector of reflectance measurements $\boldsymbol{\rho}$ and smaller dimensioned matrices $\mathbf{K}$. With these smaller datasets we then estimated the kernel weights using the GSVD or the Laplace approaches.

\subsection{Software Utilised}

We utilised R (R Core Team 2014) and RStudio (RStudio Team 2015) for data processing and analysis. Specific R packages included tidyverse (http://tidyverse. tidyverse.org) for data processing and visualization, geigen (https://CRAN . $R$-project.org/package=geigen) for implementation of the GSVD, and greybox (https://CRAN.R-project.org/package=greybox) for the Laplace parameter estimation.

\section{Results}

We present results for six sites from Table 1, selected based according to the number of reflectance measurements available for analysis. From Figure 2 three of the sites (AU-Lox, IT-CA1, and ZM-Mon) have a high number of reflectance measurements across 2017; the remaining three (JP-MPF, DE-Hai, US-Wi3) do not. Results from the sites not shown here can be found via an interactive applet (https: //jmzobitz.shinyapps.io/BRDF-shiny/). Figure 4 shows the inversion of the kernel weights (isotropic, volumetric, and geometric) for both MODIS bands 1 and 2, presented along with the corresponding BRDF kernel weights provided by the MCD43A1 
data product.

[Figure 4 about here.]

Figure 5 compares the computational runtime for GSVD method to the Tikhonov smoothing in Equation (4). We selected the matrices $\mathbf{K}, \mathbf{B}$, and vector $\boldsymbol{\rho}$ from the site AU-Lox and reflectance band 2 and evaluated our GSVD algorithm compared to an iterative algorithm that solves Equation (4) through inversion of Equation (5), applying the same bisection method to determine $\lambda$.

[Figure 5 about here.]

Figure 6 compares the calculated white sky albedo for our GSVD method to that provided by the MCD43A3 data product.

[Figure 6 about here.]

Figures 4 and 6 allow for two different comparisons of the GSVD estimates of $\boldsymbol{f}$, forward modeled reflectance $\boldsymbol{\rho}$ (computed from $\mathbf{K}$ with the GSVD estimates of $\boldsymbol{f}$ ), and white-sky albedo to the MODIS data products. We utilize a Taylor diagram (Taylor 2001) to display this variability across all of the sites and MODIS bands analyzed (Figure 7 for the BRDF kernel weights, Figure 8 for the forward modeled $\boldsymbol{\rho}$, and Figure 9 for the white sky albedo). Figure 8 additionally compares measured to modeled $\rho$ in a 1:1 plot. A Taylor diagram is displayed on a polar coordinate plot with the radius equal to the modeled standard deviation (in this case the GSVD estimates of kernel weights, reflectance, or albedo). For comparison, the horizontal axis of the Taylor diagram contains the standard deviation of the reference values (in this case the MODIS data products). The azimuthal angle in the Taylor diagram is the correlation coefficient $r$ between the modeled (GSVD) to reference (MODIS) values. In addition, the centered root mean square difference between modeled to measured values can be inferred with isoclines that are concentric circles centered at the reference standard deviation. For ease of comparison with several different sites, the quantities shown on a Taylor diagram are normalised by the reference standard deviation.

[Figure 7 about here.]

[Figure 8 about here.]

[Figure 9 about here.]

Figure 10 shows the comparison to GSVD and Laplace kernel weights from randomly subsampled reflectance data (Section 2.5). The same input data was utilized in the both inversion approaches. Figure 10 suggests there is less variability in the GSVD kernel weights even as the number of reflectance measurements decreases. A likely explanation for this is due to the influence of the smoothing matrix $\mathbf{B}$ on the inversion.

[Figure 10 about here.]

Figure 11 shows the distribution of the computed values of $\lambda$ for each of the different sites across the different MODIS bands. Generally speaking for five of the MODIS bands the distribution of $\lambda$ was uniform across the different sites. The mean value of $\lambda$ shown in Figure 11 increases as wavelength decreases, with band 1 (blue) and 2 (green) having notably higher values. A likely explanation for this is that the data in the shorter wavelengths is more affected by residual noise from aerosols. 
[Figure 11 about here.]

\section{Discussion}

\subsection{Evaluation of the GSVD algorithm compared to the MCD43 products}

Our manuscript describes and validates an algorithm to produce efficient and consistent retrievals of the MODIS MCD43 product to a specified degree of accuracy. Similar to Quaife and Lewis (2010) the value of the smoothness parameter $\lambda$ is determined by the RMSE of each waveband, but for this study we have developed an iterative method to determine $\lambda$ as part of the solution. Our approach applies a constrained least squares problem (rather than a weighted inversion as in the current operational algorithm); additional constraints can be implemented for future development of the code. Our GSVD method is objectively better than the current operational algorithm for three primary reasons.

First, our results are based on a strict set of filtering criteria which in most cases reproduced patterns in the kernel weights and albedo products. Patterns in the kernel weights and white sky albedo from the GSVD approach generally showed consistency with expected phenological patterns from deciduous broad-leaf forests, i.e. a signal that corresponds to timing of leaf-on and leaf-off depending on the timing of the season and its geographic location (Figures 4 and 6), but can be further compared using the Taylor diagrams.

We would expect some differences between the GSVD-estimated kernel weights to the MCD43A1 data products due to the addition of the smoothness matrix $\mathbf{B}$ (Figure 4). Taylor diagrams (Figures 7, 8, and 9) also provide a comprehensive comparison beyond timeseries comparisons. In Taylor diagrams both the angle and radius are important. Points that lie close to the horizontal axis with a normalised radius of unity indicate a high degree of correlation with the modeled standard deviation commensurate to the reference value. There is a high degree of correlation for the modeled reflectances and albedo data products, but less so with the kernel weights (Figure 7). The standard deviation of the kernel weights derived by our GSVD algorithm was significantly less than the standard deviation for the MCD43A1 product, which we attribute to the value of $\lambda$ generating results smoother in time than the MCD43 operational algorithm. The reduced standard deviation is consistent across all sites, except for a small number of instances in bands 5, 6 and 7. Despite this, our albedo estimates agree relatively closely with those from MCD43 albedo, with the largest deviation coming from sites with the fewest number of observations.

Second, the GSVD algorithm produced less variability in the BRDF kernel weights as the number of reflectance data used to estimate kernel weights decreases (Figure 10), and more closely align with the patterns in the MCD43A1 product. From these results we infer that the Tikhonov smoothing method used produces temporally consistent estimates of the kernel weights (and other products derived from BRDF weights) even when the number of input reflectance data decreases. Generally speaking, consistency in reproducing a data product (e.g. kernel weights, modeled reflectances, or albedo) across sites is a direct function of the number of reflectance measurements used in the inversion, as determined by the applied QA filtering (Figure 2).

Third, our forward modeled reflectances also are consistent with measured reflectance compared to the MCD43A1 data product. The Taylor diagram comparing 
modeled reflectance (Figure 8) from the GSVD approach (salmon circles) have several sites that are close to the reference value $(r=1$ with $\theta=0)$ compared the current MCD43 algorithm (blue triangles). When a point is close to the reference value on the Taylor diagram it indicates modeled values that are consistent in pattern to the reference value (in the case of Figure 8 the reference values are measured reflectances). The apparent consistency using the GSVD approach is present with modeled albedo (Figure 9) as well. From these figures we can infer that our retrievals will do a better job of estimating albedo and normalising data to specified geometries - i.e. the two primary roles that MCD43 has in the onward MODIS processing chain.

\subsection{Evaluation of the GSVD algorithm}

The algorithm presented here presents an efficient way to implement Tikhonov smoothing. Usually the value of $\lambda$ is selected at the corner of the L-curve in Figure 5 (Hansen 1992; Hansen and O'Leary 1993). For this study the value of $\lambda$ is determined by the residual norm $(=\sqrt{n} \delta)$, similar to Morozov's discrepancy principle (Hansen 1992, 1996). Our approach fixes $\delta$ to be the target theoretical accuracy (Table 2); increasing $\delta$ would make the kernel weights $\boldsymbol{f}$ more smooth.

Figure 5 illustrates that iteratively determining the value of $\lambda$ via matrix inversion of Equation (5) is computationally inefficient over large time domains. For one site and band the GSVD method is half the computational time to the Tikhonov smoothing approach. For the GSVD method a single decomposition can be done for a specified time period and then applied across different MODIS bands, rather than a separate matrix inversion for each MODIS band with the Tikhonov smoothing approach. Additional computational efficiency is gained as the residual norm is a linear combination of the GSVD decomposed matrices, facilitating selection of $\lambda$.

The efficiency of our algorithm hinges on the ability to compute the Generalised Singular Value Decomposition, which is dependent on the matrix dimensions $\mathbf{K}$ and B. It is known that computation of the GSVD scales on same order of magnitude as matrix inversion $\left(O\left(n^{3}\right)\right.$, with $n$ being the number of rows of $\mathbf{K}$, see Paige (1986); Bai (1992)). While this study concentrated over the course of a year, the window can be shortened to smaller time intervals (such as a quarter or half year) to speed processing. In addition techniques such as multiparameter Tikhonov regularization (Belge, Kilmer, and Miller 2002; Chung and Español 2017) or Krylov subspace recycling (Renaut 1998; Renaut, Lin, and Guo 2012) have the potential to expand this approach for flexibility in determining $\lambda$. Given the consistency of $\lambda$ across different MODIS bands (Figure 11), an approximate value of $\lambda$ would also provide approximate inversions rather than doing the GSVD. Future work can evaluate if this holds true across different landscapes.

The kernel weights should be non-negative based on physical considerations and semi-orthogonality of the BRDF kernels (Lucht and Schaaf 2000). In the current MCD43 algorithm this is accomplished through setting the corresponding kernel weight to zero and than re-estimating the kernel weights. The GSVD approach implemented here allows for negative kernel weights; we do not see negative kernel weights as a limitation of our method for two primary reasons.

First, the occurrence of negative kernel weights with the GSVD approach is proportionally less than what we would find for the Laplace implementation. There are four cases to consider when comparing the GSVD to the Laplace approach in terms of positive and negative kernel weights. Through a direct comparison of the two approaches across each site, band, and time (Figure 12), the GSVD approach produces 
fewer cases of negative kernel weights, and can also generate positive kernel weights when the corresponding Laplace inversion is negative. By association we would expect a similar comparison between our GSVD method to the MCD43A1 algorithm.

[Figure 12 about here.]

Second, the occurrence of negative kernel weights is related to the design of $\mathbf{B}$ and can be viewed as a trade-off between a solution (Equation (3) constrained to the observations $\mathbf{K} \boldsymbol{f}=\boldsymbol{\rho}$ or to a minimum norm solution $\|\mathbf{B} \boldsymbol{f}\|$, where the solution is expressed as a curve parameterised by $\lambda$. As $\lambda$ grows without bound Equation (3) forces the un-regularised solution $\boldsymbol{f}$ to exist in the nullspace of $\mathbf{B}$. Since the matrix $\mathbf{B}$ consists of first order differences across time, the nullspace for $\mathbf{B}$ contains entirely constant vectors. Through the GSVD transformations that generate a solution $\boldsymbol{f}$ (detailed in the Supplemental data), one of these constant vectors is negative.

Future refinements to our algorithm can be applied in two approaches. First, a nonnegativity constraint can be incorporated directly into Equation (4) as a constrained least squares problem. Constrained least squares have separate algorithms for computation (Golub 1973; Bro and De Jong 1997; Chen and Plemmons 2009; Golub and Van Loan 2014); to the best of our knowledge no study has combined the GSVD approach with a constrained least squares optimization with non-negativity constraints. Second, Equation (4) could be defined as minimizing $\|\mathbf{K} \boldsymbol{f}-\boldsymbol{\rho}\|$ subject to $\|\mathbf{B} \boldsymbol{f}\|=0$. This alternative least squares problem is mathematically equivalent to determining an $f$ in the nullspace of $\mathbf{B}$ that also minimizes the residual norm. Such an approach could be a more tractable problem to solve because the space of potential solutions for $f$ is confined to the nullspace of $\mathbf{B}$.

\section{Conclusions}

We have described a new algorithm for the inversion of linear kernel-driven BRDF models that allows for retrievals to be performed over arbitrary length time windows, where the values of the kernel weights can vary within that window and in cases where data is relatively sparse. Our approach builds on that of Quaife and Lewis (2010) but includes an efficient automated method for determining the strength of the smoothness constraint in the inversion. Although the kernel weights retrieved by our method are notably different from those in the MCD43 product the values of albedo and reflectance calculated from these are similar to those in MCD43. In particular we find that the surface reflectance values derived by our method match more closely with data from the MODIS surface reflectance product than those forward modelled from MCD43 parameters.

\section{Acknowledgments}

JMZ was supported through an IES Abroad Research Grant and an Augsburg University Provost Research Grant. JMZ would like to thank B. N. Acheson for helpful discussions on this manuscript. TQ and NKN were supported in part by the UK Natural Environmental Research Council National Centre for Earth Observation (NE/R016518/1). 


\section{References}

Anderson, E., Z. Bai, C. Bischof, S. Blackford, J. Demmel, J. Dongarra, J. Du Croz, et al. 1999. LAPACK Users' Guide. 3rd ed. Philadelphia, PA: Society for Industrial and Applied Mathematics.

AppEEARS Team. 2018. "Application for Extracting and Exploring Analysis Ready Samples (AppEEARS). Ver. 2.14.2." https://lpdaacsvc.cr.usgs.gov/appeears.

Bai, Zhaojun. 1992. "The CSD, GSVD, Their Applications and Computations." In IMA Preprints Series, Vol. 958, 3-5. University of Minnesota.

Belge, Murat, Misha E. Kilmer, and Eric L. Miller. 2002. "Efficient Determination of Multiple Regularization Parameters in a Generalized L-Curve Framework." Inverse Problems 18 (4): 1161-1183.

Bro, Rasmus, and Sijmen De Jong. 1997. "A Fast Non-Negativity-Constrained Least Squares Algorithm." Journal of Chemometrics 11 (5): 393-401.

Chen, Donghui, and Robert J. Plemmons. 2009. "Nonnegativity Constraints in Numerical Analysis." In The Birth of Numerical Analysis, 109-139. World Scientific.

Chen, JM, and J Cihlar. 1997. "A hotspot function in a simple bidirectional reflectance model for satellite applications." Journal of Geophysical Research: Atmospheres 102 (D22): 2590725913.

Chung, Julianne, and Malena I. Español. 2017. "Learning Regularization Parameters for General-Form Tikhonov." Inverse Problems 33 (7): 074004.

Cleugh, Helen A., Ray Leuning, Qiaozhen Mu, and Steven W. Running. 2017. "Regional Evaporation Estimates from Flux Tower and MODIS Satellite Data." Remote Sensing of Environment 106 (3): 285-304.

Gao, Feng, Xiaowen Li, Alan Strahler, and Crystal Schaaf. 2000. "Evaluation of the Li transit kernel for BRDF modeling." Remote Sensing Reviews 19 (1-4): 205-224.

Garonna, Irene, Rogier de Jong, Allard J.W. de Wit, Caspar A. Mücher, Bernhard Schmid, and Michael E. Schaepman. 2014. "Strong Contribution of Autumn Phenology to Changes in Satellite-Derived Growing Season Length Estimates across Europe (1982-2011)." Global Change Biology 20 (11): 3457-3470.

Golub, Gene H. 1973. "Some Modified Matrix Eigenvalue Problems." SIAM Review 15 (2): 318-334.

Golub, Gene H., and C. Van Loan. 2014. Matrix Computations. 4th ed. Johns Hopkins University Press.

Guay, Kevin C., Pieter S. A. Beck, Logan T. Berner, Scott J. Goetz, Alessandro Baccini, and Wolfgang Buermann. 2014. "Vegetation Productivity Patterns at High Northern Latitudes: A Multi-Sensor Satellite Data Assessment." Global Change Biology 20 (10): 3147-3158.

Hansen, P. 1992. "Analysis of Discrete Ill-Posed Problems by Means of the L-Curve." SIAM Review 34 (4): 561-580.

Hansen, P., and D. O'Leary. 1993. "The Use of the L-Curve in the Regularization of Discrete Ill-Posed Problems." SIAM Journal on Scientific Computing 14 (6): 1487-1503.

Hansen, Per Christian. 1996. Rank-Deficient and Discrete Ill-Posed Problems: Numerical Aspects of Linear Inversion. SIAM Books, Philadelphia, PA.

Hashimoto, Hirofumi, Jennifer L. Dungan, Michael A. White, Feihua Yang, Andrew R. Michaelis, Steven W. Running, and Ramakrishna R. Nemani. 2008. "Satellite-Based Estimation of Surface Vapor Pressure Deficits Using MODIS Land Surface Temperature Data." Remote Sensing of Environment 112 (1): 142-155.

Hilker, Thomas, Nicholas C. Coops, Michael A. Wulder, T. Andrew Black, and Robert D. Guy. 2008. "The Use of Remote Sensing in Light Use Efficiency Based Models of Gross Primary Production: A Review of Current Status and Future Requirements." Science of The Total Environment 404 (2-3): 411-423.

Hirschmugl, Manuela, Heinz Gallaun, Matthias Dees, Pawan Datta, Janik Deutscher, Nikos Koutsias, and Mathias Schardt. 2017. "Methods for Mapping Forest Disturbance and Degradation from Optical Earth Observation Data: A Review." Current Forestry Reports 3 (1): 
$32-45$.

Hufkens, Koen, Mark Friedl, Oliver Sonnentag, Bobby H. Braswell, Thomas Milliman, and Andrew D. Richardson. 2012. "Linking Near-Surface and Satellite Remote Sensing Measurements of Deciduous Broadleaf Forest Phenology." Remote Sensing of Environment 117: $307-321$.

Jiao, Ziti, Anxin Ding, Alexander Kokhanovsky, Crystal Schaaf, Francois-Marie Bréon, Yadong Dong, Zhuosen Wang, et al. 2019. "Development of a snow kernel to better model the anisotropic reflectance of pure snow in a kernel-driven BRDF model framework." Remote sensing of environment 221: 198-209.

Jiao, Ziti, Crystal B Schaaf, Yadong Dong, Miguel Román, Michael J Hill, Jing M Chen, Zhuosen Wang, et al. 2016. "A method for improving hotspot directional signatures in BRDF models used for MODIS." Remote Sensing of Environment 186: 135-151.

Kansakar, Pratistha, and Faisal Hossain. 2016. "A Review of Applications of Satellite Earth Observation Data for Global Societal Benefit and Stewardship of Planet Earth." Space Policy 36: 46-54.

Kussul, N., S. Skakun, A. Shelestov, M. Lavreniuk, B. Yailymov, and O. Kussul. 2015. "Regional Scale Crop Mapping Using Multi-Temporal Satellite Imagery." In ISPRS - International Archives of the Photogrammetry, Remote Sensing and Spatial Information Sciences, Vol. XL-7/W3, Berlin, Germany, 45-52.

Li, Zhao-Liang, Bo-Hui Tang, Hua Wu, Huazhong Ren, Guangjian Yan, Zhengming Wan, Isabel F. Trigo, and José A. Sobrino. 2013. "Satellite-Derived Land Surface Temperature: Current Status and Perspectives." Remote Sensing of Environment 131: 14-37.

Lucht, Wolfgang, and Crystal Barker Schaaf. 2000. "An Algorithm for the Retrieval of Albedo from Space Using Semiempirical BRDF Models." IEEE Transactions on Geoscience and Remote Sensing 38 (2): 977-998.

Mahecha, Miguel D., Fabian Gans, Sebastian Sippel, Jonathan F. Donges, Thomas Kaminski, Stefan Metzger, Mirco Migliavacca, Dario Papale, Anja Rammig, and Jakob Zscheischler. 2017. "Detecting Impacts of Extreme Events with Ecological in Situ Monitoring Networks." Biogeosciences 14 (18): 4255-4277.

Mu, Qiaozhen, Maosheng Zhao, John S. Kimball, Nathan G. McDowell, and Steven W. Running. 2013. "A Remotely Sensed Global Terrestrial Drought Severity Index." Bulletin of the American Meteorological Society 94 (1): 83-98.

Paige, C. C. 1986. "Computing the Generalized Singular Value Decomposition." SIAM Journal on Scientific and Statistical Computing 7 (4): 1126-1146.

Pan, Shufen, Hanqin Tian, Shree R. S. Dangal, Zhiyun Ouyang, Bo Tao, Wei Ren, Chaoqun Lu, and Steven Running. 2014. "Modeling and Monitoring Terrestrial Primary Production in a Changing Global Environment: Toward a Multiscale Synthesis of Observation and Simulation." Advances in Meteorology .

Qu, Ying, Qiang Liu, Shunlin Liang, Lizhao Wang, Nanfeng Liu, and Suhong Liu. 2014. "Direct-Estimation Algorithm for Mapping Daily Land-Surface Broadband Albedo From MODIS Data." IEEE Transactions on Geoscience and Remote Sensing 52 (2): 907-919.

Quaife, T., and P. Lewis. 2010. "Temporal Constraints on Linear BRDF Model Parameters." IEEE Transactions on Geoscience and Remote Sensing 48 (5): 2445-2450.

R Core Team. 2014. R: A Language and Environment for Statistical Computing. Vienna, Austria: R Foundation for Statistical Computing.

Renaut, Rosemary A. 1998. "A Parallel Multisplitting Solution of the Least Squares Problem." Numerical linear algebra with applications 5 (1): 11-31.

Renaut, Rosemary A., Youzuo Lin, and Hongbin Guo. 2012. "Multisplitting for Regularized Least Squares with Krylov Subspace Recycling." Numerical Linear Algebra with Applications 19 (4): 655-676.

Roujean, Jean-Louis, Marc Leroy, and Pierre-Yves Deschamps. 1992. "A Bidirectional Reflectance Model of the Earth's Surface for the Correction of Remote Sensing Data." Journal of Geophysical Research 97 (D18): 20455.

RStudio Team. 2015. RStudio: Integrated Development Environment for R. Boston, MA: RStu- 
dio, Inc.

Schaaf, Crystal Barker, and Zongming Wang. 2018a. "MCD43A1 MODIS/Terra+Aqua BRDF/Albedo Model Parameters Daily L3 Global - 500m V006." doi: 10.5067/MODIS/MCD43A3.006. Accessed September 17, 2018.

Schaaf, Crystal Barker, and Zongming Wang. 2018b. "MCD43A3 MODIS/Terra+Aqua BRDF/Albedo Daily L3 Global - 500m V006." doi: 10.5067/MODIS/MCD43A3.006. Accessed September 17, 2018.

Soudani, Kamel, Guerric le Maire, Eric Dufrêne, Christophe François, Nicolas Delpierre, Erwin Ulrich, and Sébastien Cecchini. 2008. "Evaluation of the Onset of Green-up in Temperate Deciduous Broadleaf Forests Derived from Moderate Resolution Imaging Spectroradiometer (MODIS) Data." Remote Sensing of Environment 112 (5): 2643-2655.

Strahler, A H, and J.-P. Muller. 1999. MODIS BRDF/Albedo Product: Algorithm Theoretical Basis Document Version 5.0. Technical Report. NASA.

Taylor, Karl E. 2001. "Summarizing Multiple Aspects of Model Performance in a Single Diagram." Journal of Geophysical Research: Atmospheres 106 (D7): 7183-7192.

Tikhonov, Andre-I. Nikolaevich. 1977. Solutions of Ill Posed Problems. Washington : New York: Wiley.

Turner, David P., William D. Ritts, Warren B. Cohen, Stith T. Gower, Steve W. Running, Maosheng Zhao, Marcos H. Costa, et al. 2006. "Evaluation of MODIS NPP and GPP Products across Multiple Biomes." Remote Sensing of Environment 102 (3-4): 282-292.

Turner, David P., William D. Ritts, Warren B. Cohen, Stith T. Gower, Maosheng Zhao, Steve W. Running, Steven C. Wofsy, Shawn Urbanski, Allison L. Dunn, and J. W. Munger. 2003. "Scaling Gross Primary Production (GPP) over Boreal and Deciduous Forest Landscapes in Support of MODIS GPP Product Validation." Remote Sensing of Environment 88 (3): $256-270$.

Vermote, E., and R. Wolfe. 2018a. "MOD09GA MODIS/Terra Surface Reflectance Daily L2G Global 1kmand 500m SIN Grid V006 [Data Set]." doi: 10.5067/MODIS/MOD09GA.006. Accessed September 17, 2018.

Vermote, E., and R. Wolfe. 2018b. "MYD09GA MODIS/Aqua Surface Reflectance Daily L2G Global 1km and 500m SIN Grid V006 [Data Set]." doi: 10.5067/MODIS/MYD09GA.006. Accessed September 17, 2018.

Wang, Zhuosen, Crystal B. Schaaf, Qingsong Sun, Yanmin Shuai, and Miguel O. Román. 2018. "Capturing Rapid Land Surface Dynamics with Collection V006 MODIS BRDF/NBAR/Albedo (MCD43) Products." Remote Sensing of Environment 207: 50-64.

Wanner, W., A. H. Strahler, B. Hu, P. Lewis, J.-P. Muller, X. Li, C. L. Barker Schaaf, and M. J. Barnsley. 1997. "Global Retrieval of Bidirectional Reflectance and Albedo over Land from EOS MODIS and MISR Data: Theory and Algorithm." Journal of Geophysical Research: Atmospheres 102 (D14): 17143-17161. 
List of Tables 


\begin{tabular}{|c|c|c|c|c|}
\hline Site ID & Latitude $\left(^{\circ}\right)$ & Longitude $\left(^{\circ}\right)$ & Location & Fluxnet DOI \\
\hline AU-Lox & -34.4704 & 140.6551 & Loxton & DOI $: 10.18140 /$ FLX/1440247 \\
\hline $\mathrm{CA}-\mathrm{Oas}$ & 53.6289 & -106.1978 & Saskatchewan - Western Boreal Mature Aspen & DOI $: 10.18140 /$ FLX $/ 1440043$ \\
\hline CA-TPD & 42.6353 & -80.5577 & Ontario - Turkey Point Mature Deciduous & DOI $: 10.18140 /$ FLX/1440112 \\
\hline DE-Hai & 51.0792 & 10.453 & Hainich & DOI $: 10.18140 /$ FLX/1440148 \\
\hline DE-Lnf & 51.3282 & 10.3678 & Leinefelde & DOI $: 10.18140 /$ FLX/1440150 \\
\hline DK-Sor & 55.4859 & 11.6446 & Soroe & DOI $: 10.18140 /$ FLX/1440155 \\
\hline FR-Fon & 48.4764 & 2.7801 & Fontainebleau-Barbeau & DOI : $10.18140 /$ FLX/1440161 \\
\hline IT-CA1 & 42.3804 & 12.0266 & Castel d'Asso1 & DOI : $10.18140 /$ FLX/1440230 \\
\hline IT-CA3 & 42.38 & 12.0222 & Castel d'Asso 3 & DOI : $10.18140 / F L X / 1440232$ \\
\hline IT-Col & 41.8494 & 13.5881 & Collelongo & DOI $: 10.18140 /$ FLX/1440167 \\
\hline IT-Isp & 45.8126 & 8.6336 & Ispra ABC-IS & DOI : $10.18140 /$ FLX/1440234 \\
\hline IT-PT1 & 45.2009 & 9.061 & Parco Ticino forest & DOI : $10.18140 /$ FLX/1440172 \\
\hline IT-Ro1 & 42.4081 & 11.93 & Roccarespampani 1 & DOI $: 10.18140 / F L X / 1440174$ \\
\hline IT-Ro2 & 42.3903 & 11.9209 & Roccarespampani 2 & DOI : $10.18140 /$ FLX/1440175 \\
\hline JP-MBF & 44.3869 & 142.3186 & Moshiri Birch Forest Site & DOI $: 10.18140 /$ FLX/1440238 \\
\hline PA-SPn & 9.3181 & -79.6346 & Sardinilla Plantation & DOI $: 10.18140 /$ FLX/1440180 \\
\hline US-Ha1 & 42.5378 & -72.1715 & Harvard Forest EMS Tower & DOI : $10.18140 /$ FLX/1440071 \\
\hline US-MMS & 39.3232 & -86.4131 & Morgan Monroe State Forest & DOI $: 10.18140 /$ FLX/1440083 \\
\hline US-Oho & 41.5545 & -83.8438 & Oak Openings & DOI $: 10.18140 / F L X / 1440088$ \\
\hline US-UMB & 45.5598 & -84.7138 & Univ. of Mich. Biological Station & DOI $: 10.18140 / F L X / 1440093$ \\
\hline US-UMd & 45.5625 & -84.6975 & UMBS Disturbance & DOI $: 10.18140 /$ FLX/1440101 \\
\hline US-WCr & 45.8059 & -90.0799 & Willow Creek & DOI $: 10.18140 /$ FLX/1440095 \\
\hline US-Wi1 & 46.7305 & -91.2329 & Wisconsin Intermediate Hardwood & DOI : $10.18140 /$ FLX/1440054 \\
\hline US-Wi3 & 46.6347 & -91.0987 & Wisconsin Mature Hardwood & DOI : $10.18140 /$ FLX/1440057 \\
\hline US-Wi8 & 46.7223 & -91.2524 & Wisconsin Clearcut Young Hardwood & DOI $: 10.18140 /$ FLX/1440062 \\
\hline ZM-Mon & -15.4378 & 23.2528 & Mongu & DOI $: 10.18140 /$ FLX/1440189 \\
\hline
\end{tabular}

Table 1. Description of each FLUXNET site utilized in this study. All selected sites are from deciduous broadleaf forests. We selected MODIS pixels co-located with the respective eddy covariance tower monitored in the FLUXNET database. (www.fluxdata.org). Data were accessed through the AppEEARS web service (https://lpdaacsvc.cr.usgs.gov/appeears/, AppEEARS Team (2018)) 
Band

\begin{tabular}{l|lllllll}
$\delta$ (Reflectance) & 0.005 & 0.014 & 0.008 & 0.005 & 0.012 & 0.006 & 0.003
\end{tabular}

Table 2. Utilised values of $\delta$ for the GSVD algorithm each MODIS band. The algorithm proceeds to determine

an $\boldsymbol{f}$ such that the residual RMSE equals $\delta$ for each band. The $\delta$ values reflect the typical theoretical accuracy for each reflectance band (see http://modis-sr.ltdri.org/pages/validation.html for information regarding validation of the MOD09 surface reflectance product). 
List of Figures 


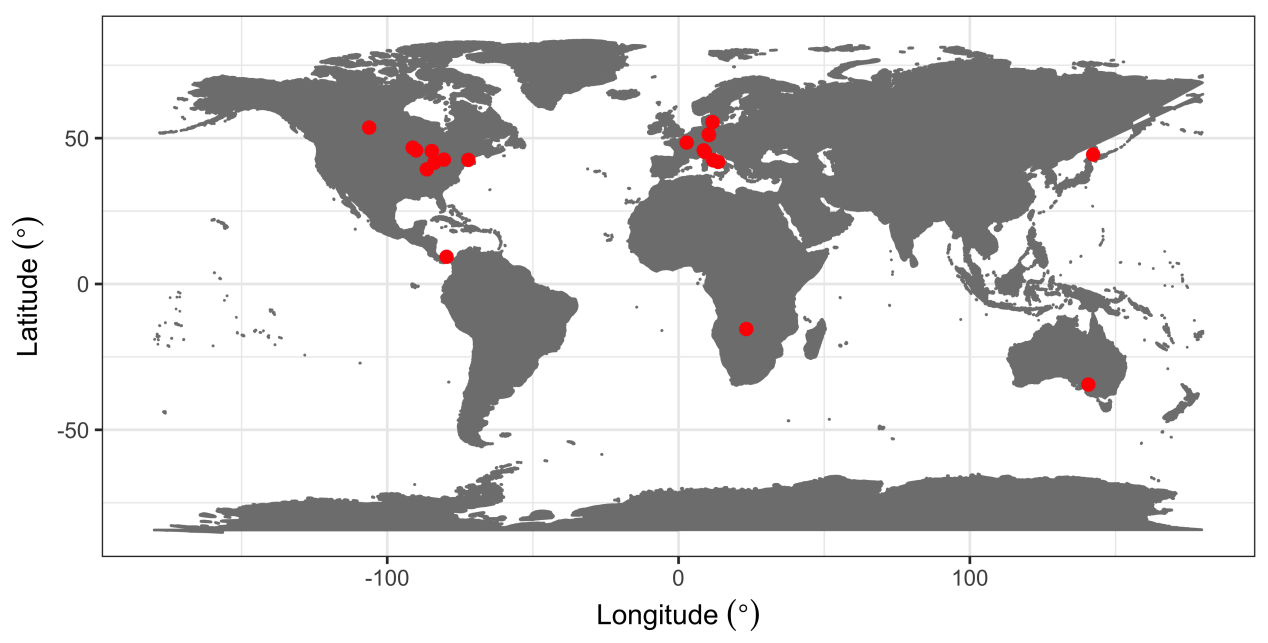

Figure 1. Geographic distribution of sites from the FLUXNET 2015 dataset for this study. 


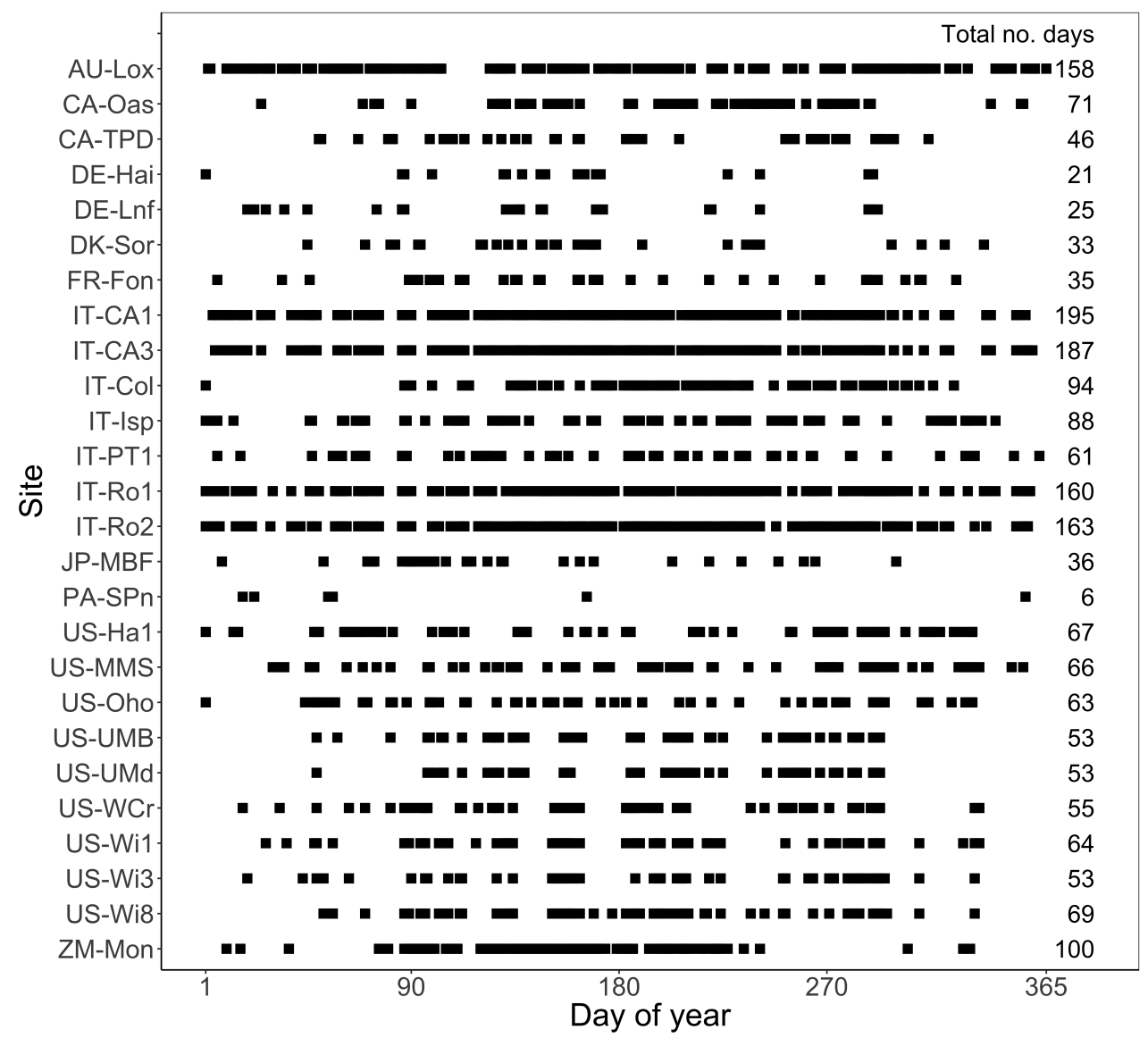

Figure 2. Distribution of the daily temporal coverage in 2017 for each of the sites listed in Table 1, where a dot represents a day where reflectance measurement $\boldsymbol{\rho}$ was utilised to determine BRDF kernel weights (Equation (3)) for each site and reflectance band using the QA filtering described in Section 2.1. The total number of unique days is listed on the right hand side of the figure. 


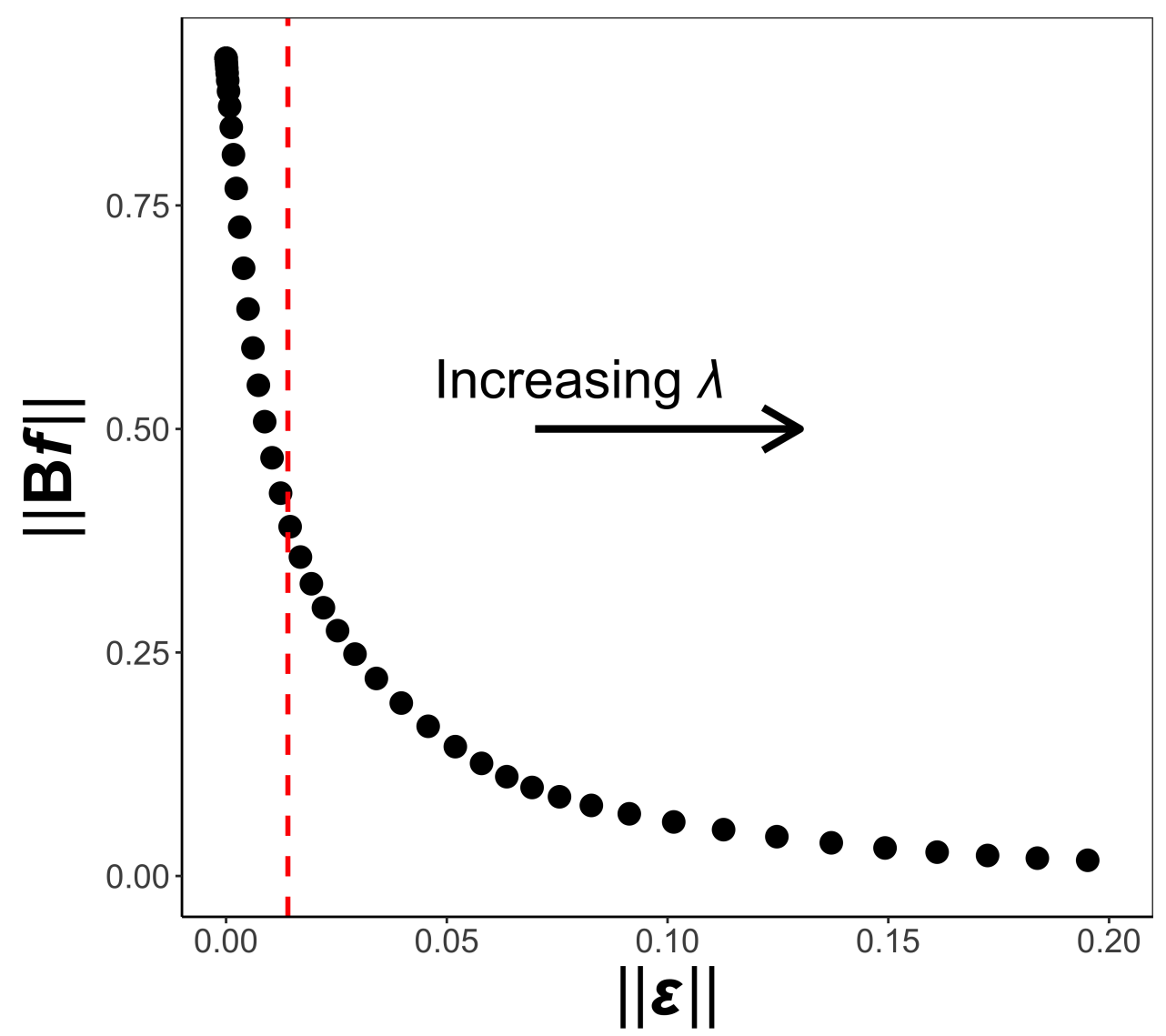

Figure 3. Sample L-curve illustrating the relationship between the solution norm $(\|\mathbf{B} \boldsymbol{f}\|)$ and the residual norm $(\|\boldsymbol{\epsilon}\|)$. The curve is parameterised by $\lambda$, which is a weighting towards a solution conditioned to be more smooth (minimizing the solution norm) or conditioned on the residual norm. 

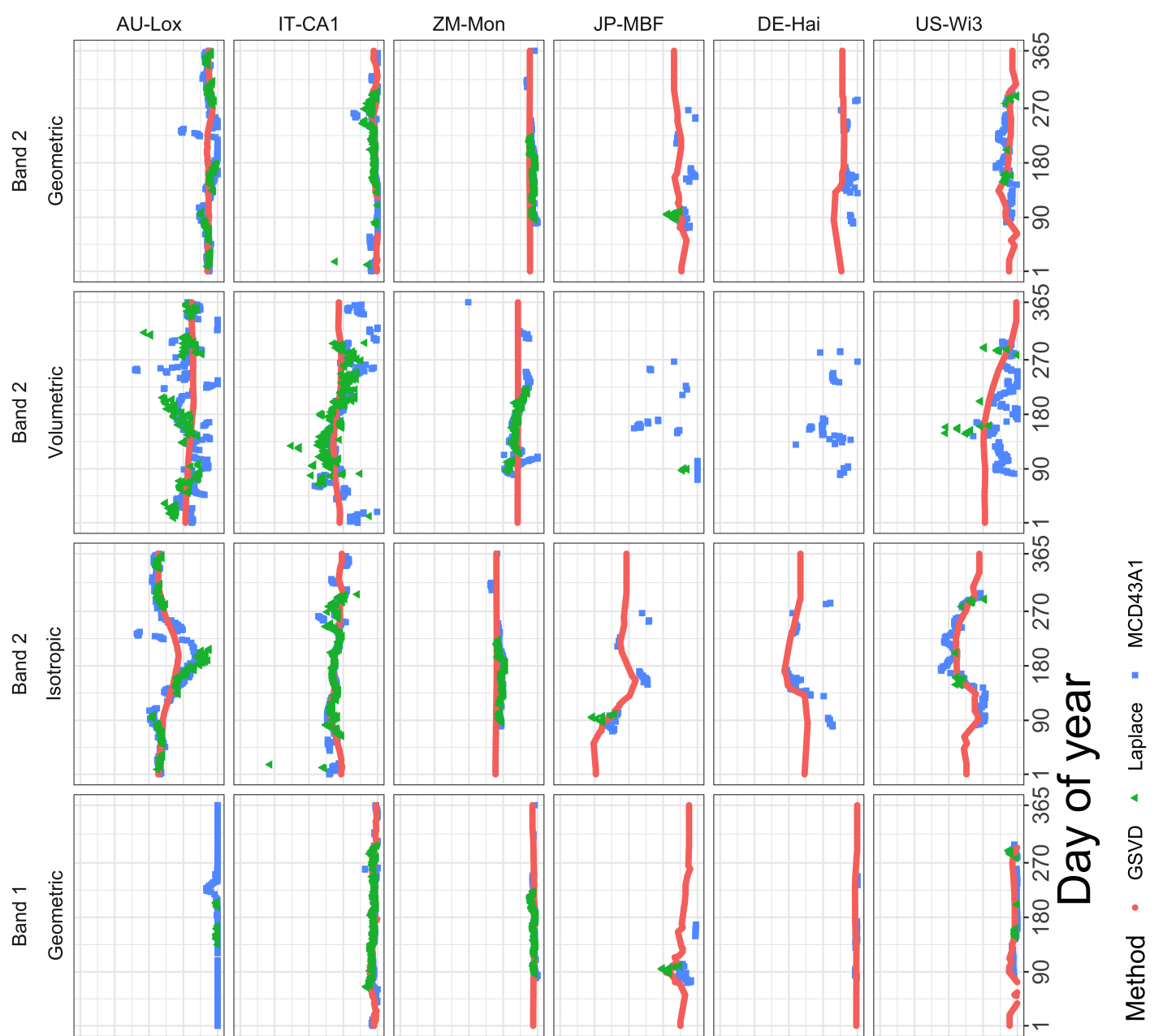
希表 总过 항 $\rightarrow$ क 莽 을

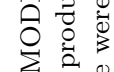
需营 政 资

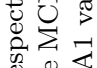
可 로월

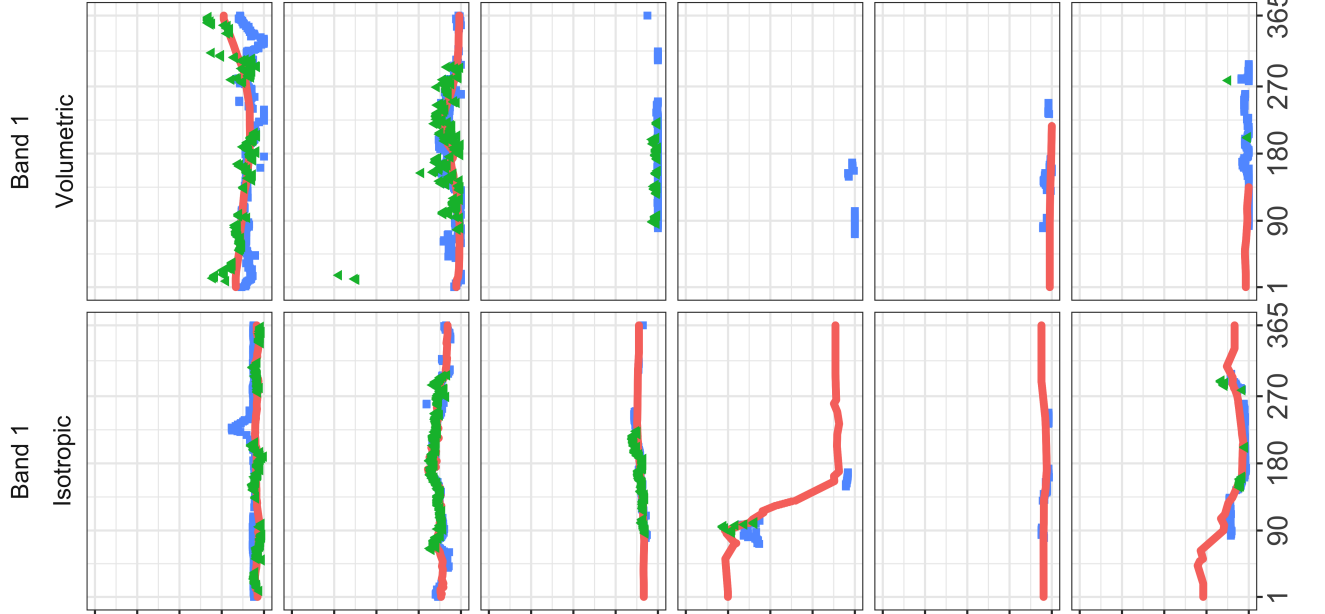

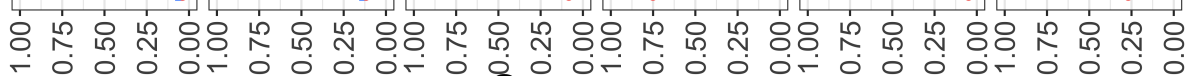

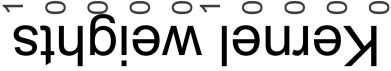

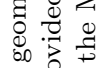
굴

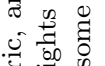
is 马 3

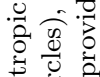

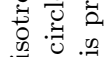

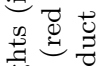

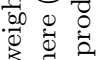
诺 渮 هi 응 응 密 


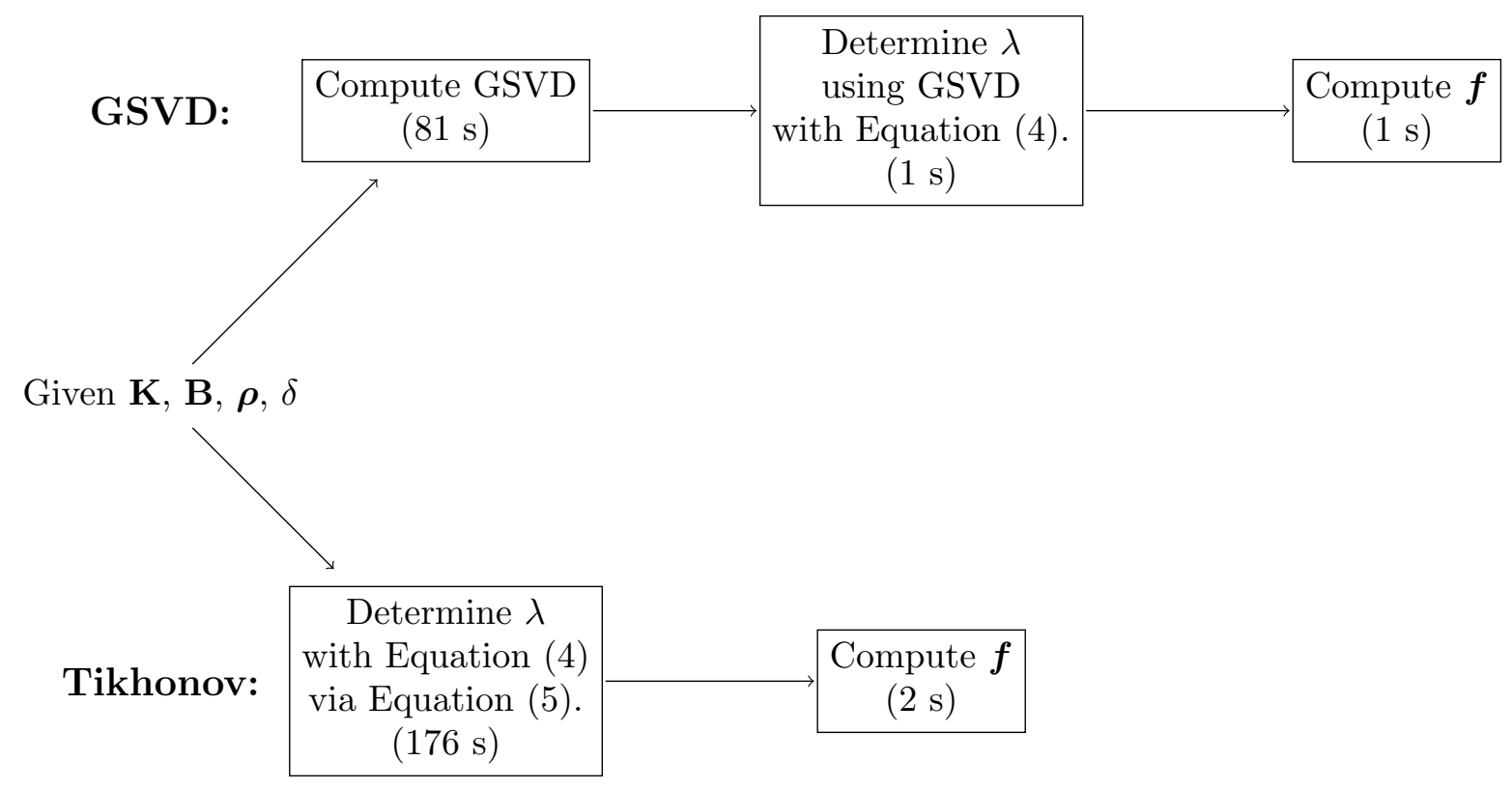

Figure 5. Runtime comparison of the runtime to solve Equation (4) via the GSVD method and the Tikhonov smoothing method (inversion of Equation (5)) using an iterative bisection method to determine $\lambda$. Runtime is presented for the site AU-Lox and Band 2. 


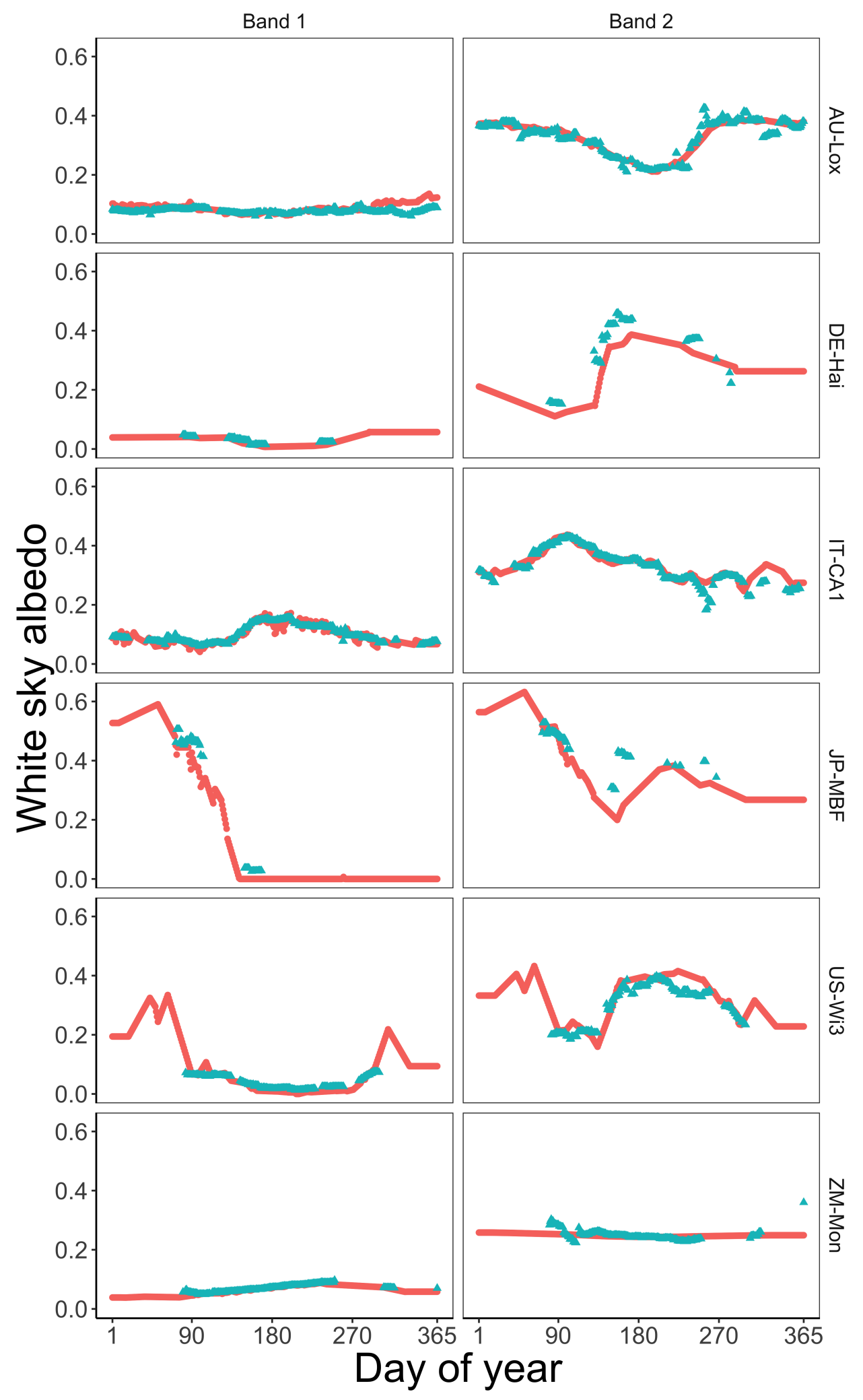

Method - GSVD $\triangle$ MCD43A3

Figure 6. Calculated albedo for MODIS bands 1 and 2 for six of the MODIS pixels analyzed, determined from the GSVD inversion presented here (red circles) or the MCD43A1 data product (blue triangles). Some of the MCD43A3 measurements for a given site were omitted by the quality assurance filtering. 


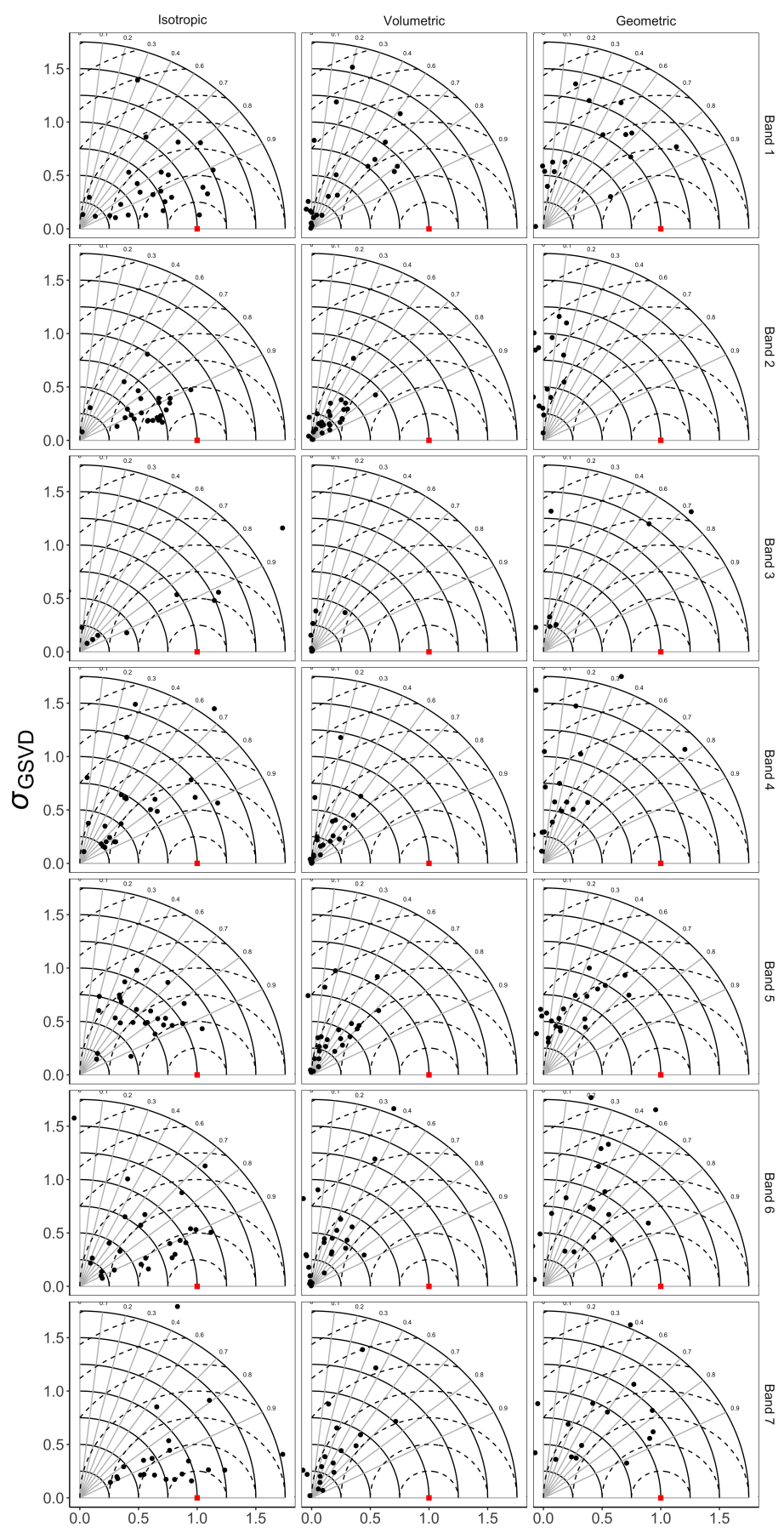

Figure 7. Taylor diagram for kernel weights (isotropic, volumetric, and geometric respectively) by the GSVD method across all seven MODIS bands and sites analyzed in this study, normalised by the reference standard deviation (MCD43A1 data product). The Taylor diagram is a polar coordinate system. The location of a point on the Taylor diagram conveys the standard deviation in the GSVD estimates, with the angle representing the correlation coefficient $r$ (radial values of $r$ annotated). The red square on the horizontal axis at $r=1$ is the normalised reference standard deviation. The concentric circles centered at the reference standard deviation allow for the inference of the centered root mean square difference between the GSVD and the MODIS data products. Each concentric circle represents centered RMS difference of 0.25. 


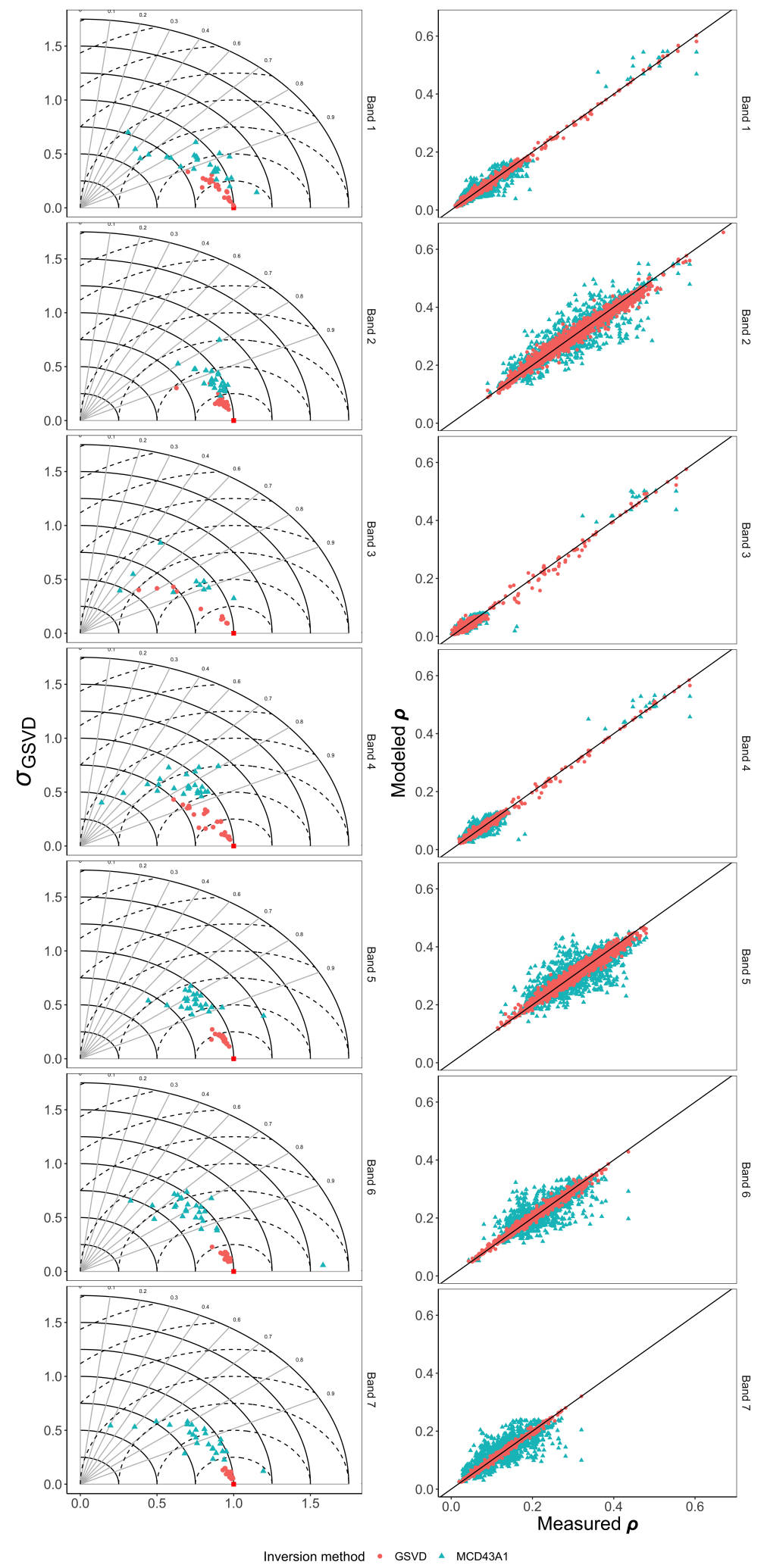

Figure 8. Left plots: Taylor diagram for the forward modeled reflectance from the GSVD method (salmon circles) or the current MCD43A1 algorithm (blue triangles) across all seven MODIS bands and sites analyzed in this study, normalized by the reference standard deviation (measured MCD43A1 reflectance). This figure has the same annotations as Figure 7. Each concentric circle represents centered RMS difference of 0.25. Right plots: Comparison of measured to modeled $\rho$ from the GSVD method (salmon circles) or the current MCD43A1 algorithm (blue triangles) across all seven MODIS bands and sites analyzed in this study. 


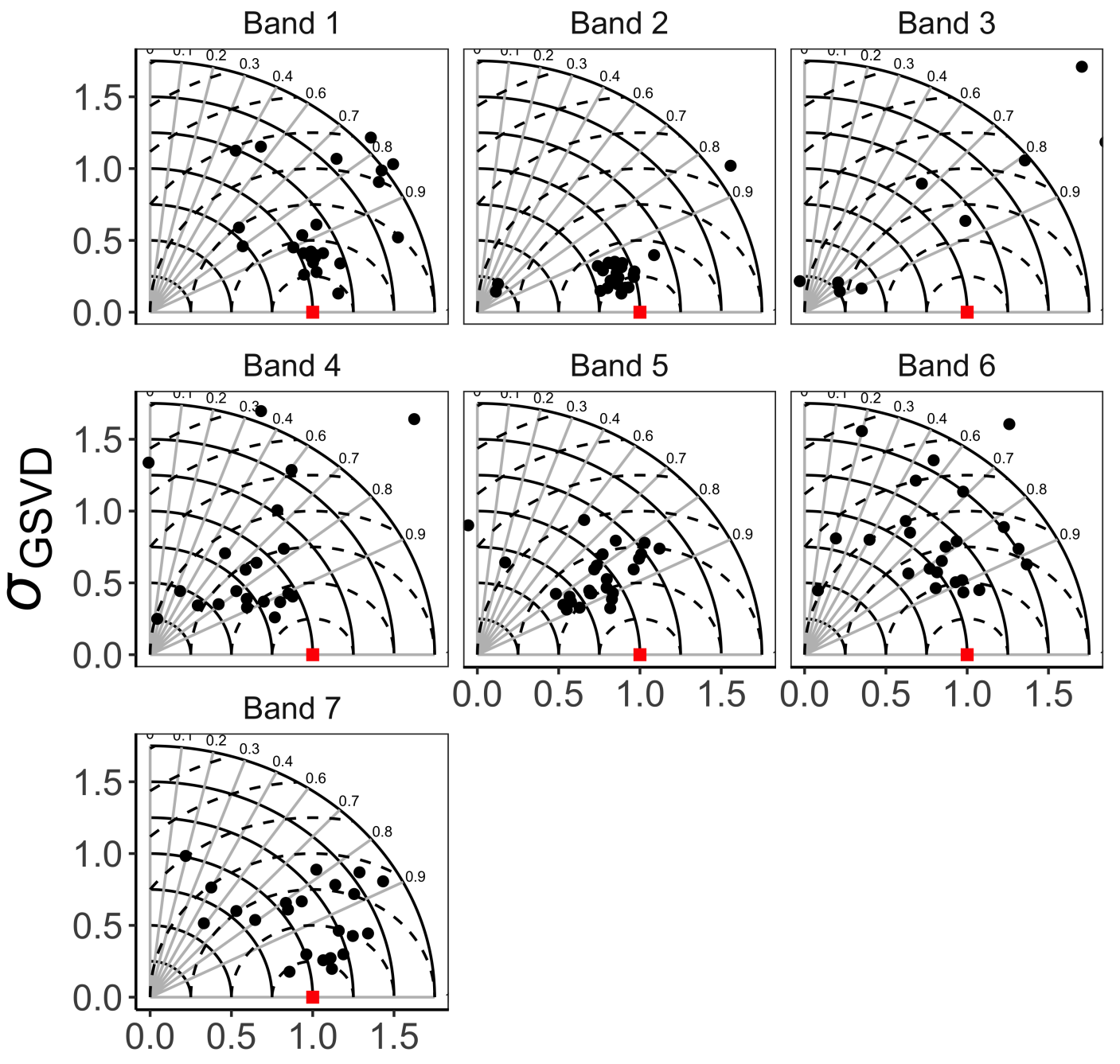

Figure 9. Taylor diagram for the white sky albedo from the GSVD method across all seven MODIS bands and sites analyzed in this study, normalised by the reference standard deviation (MCD43A3 data product). This figure has the same annotations as Figure 7. Each concentric circle represents centered RMS difference of 0.25 . 


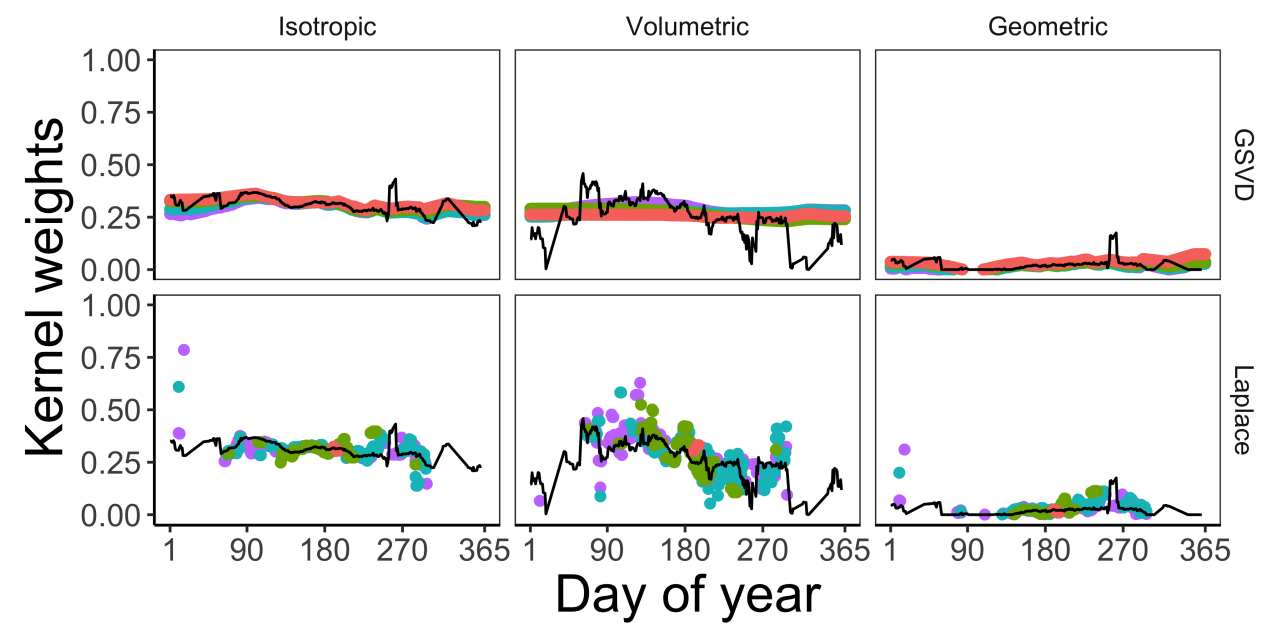

Data Utilized $\rightarrow 25 \% \rightarrow 50 \% \rightarrow 75 \% \rightarrow 100 \% \rightarrow$ MCD43A1

Figure 10. Kernel weights (isotropic, volumetric, and geometric respectively) from MODIS band 2, using sampled data from site IT-CA1. The percentages refer to the percentage of reflectance measurements subsampled. The black line is the MCD43A1 data product for comparison. 


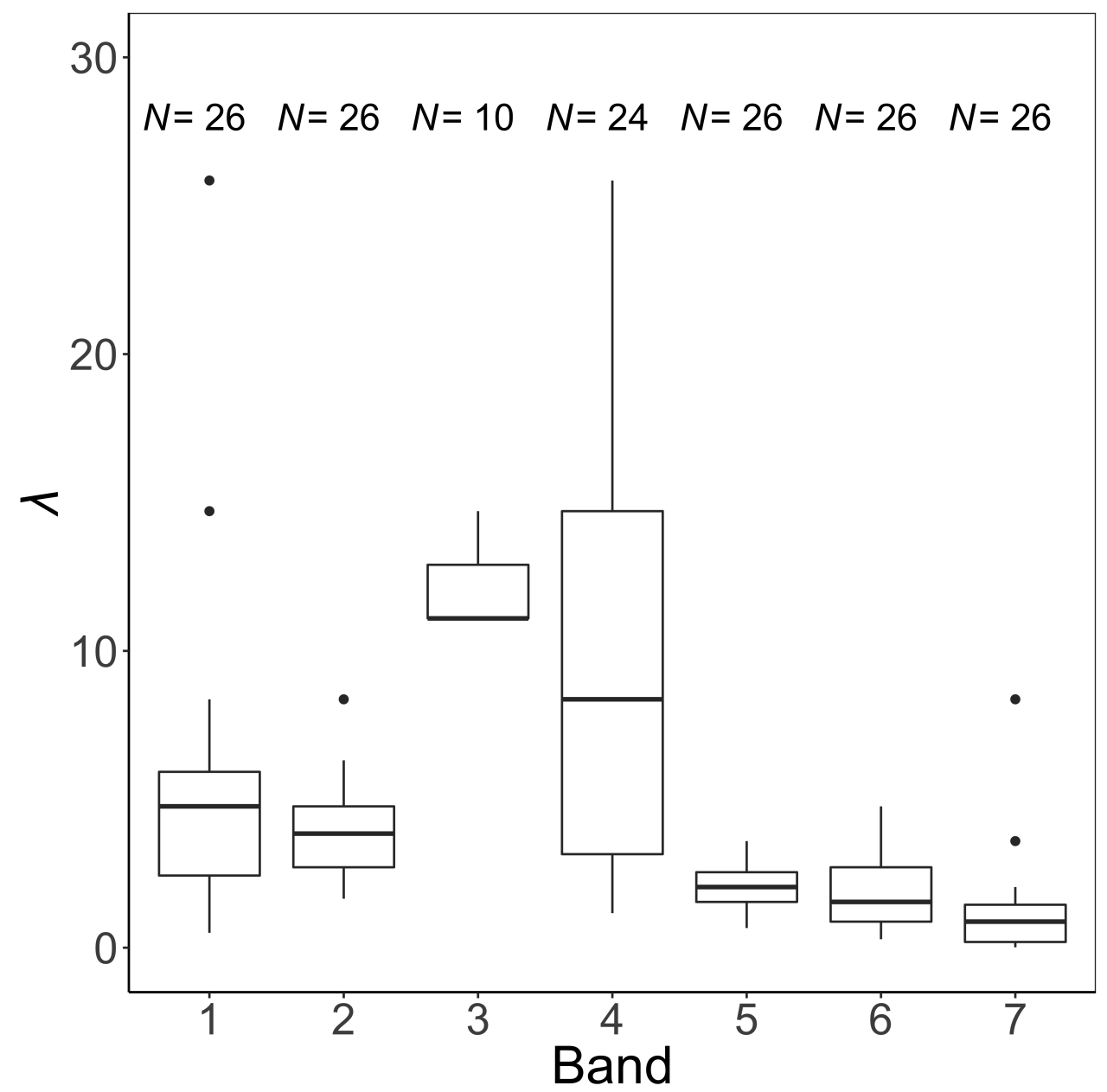

Figure 11. Distribution of optimised values of $\lambda$ for each MODIS band for the analyzed MODIS pixels. The number of sites where the GSVD method converged (out of 26) is labeled for each MODIS band. 


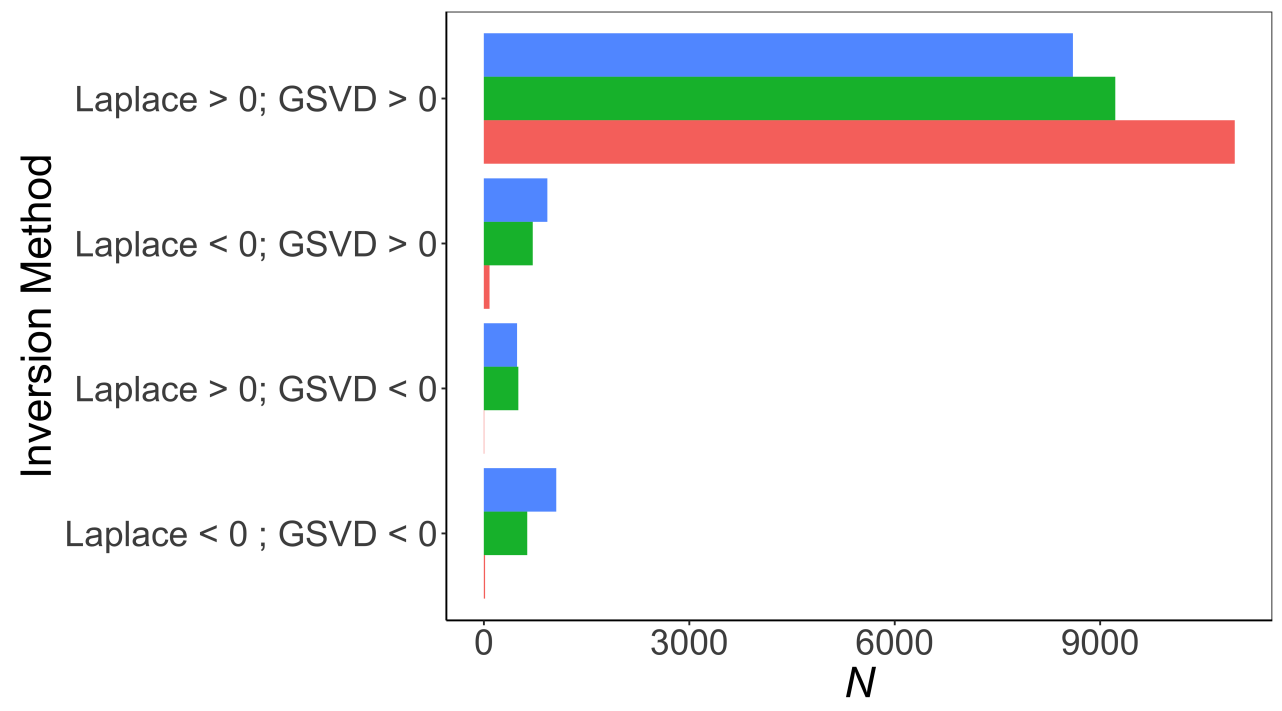

Kernel weights $\square$ Isotropic $\square$ Volumetric $\square$ Geometric

Figure 12. Comparison of negative values from the GSVD to the Laplace inversion approach, using kernel weights across all the sites, band, and days in this study. Cases are grouped compared to when the GSVD or Laplace inversion produces a positive or negative kernel weight value. 